To appear in ApJ, vol. 664

\title{
Changes in the Radio Appearance of MWC 349A
}

\author{
Luis F. Rodríguez and Yolanda Gómez \\ Centro de Radioastronomía y Astrofísica, Universidad Nacional Autónoma de México, \\ Morelia 58089, México \\ 1.rodriguez, y.gomez@astrosmo.unam.mx \\ and \\ Daniel Tafoya ${ }^{1}$ \\ Harvard-Smithsonian Center for Astrophysics, 60 Garden Street, Cambridge, MA 02138, \\ USA \\ dtafoya@cfa.harvard.edu
}

\begin{abstract}
We present new sensitive, high angular resolution 1.3, 2, and $6 \mathrm{~cm}$ observations of the continuum emission from the peculiar emission-line star MWC 349A, made with the Very Large Array. This radio emission is believed to originate in an ionized flow produced by the photoevaporation of a disk that surrounds the star. We determine for the first time the proper motion of this source, which is consistent with that expected for the location of the source in the galaxy. Our analysis of the images, that include the new observations as well as archive data covering a time interval of more than 20 years, indicates that the appearance of MWC 349A has been systematically changing over time. The well-defined "hourglass" shape that characterized the 2 and $1.3 \mathrm{~cm}$ appearance of the source in the early 1980's has disappeared to be replaced by a more "square" shape. We discuss if these changes can be accounted for by precession of the MWC 349A disk or by intrinsic changes in the parameters of the disk, but could not reach a satisfactory explanation.
\end{abstract}

Subject headings: ISM: outflows - individual (MWC 349A) - ISM: photoevaporated disks

\footnotetext{
${ }^{1}$ Centro de Radioastronomía y Astrofísica, Universidad Nacional Autónoma de México, Morelia 58089, México
} 


\section{Introduction}

MWC 349A is the brightest thermal radio continuum star in the centimeter domain (Braes, Habing, \& Schoenmaker 1972). At a distance of 1200 pc it has a bolometric luminosity of $30,000 \mathrm{~L}_{\odot}$ (Cohen et al. 1985) and exhibits an unusually slow and dense stellar wind with a velocity of $\sim 50 \mathrm{~km} \mathrm{~s}^{-1}$ (Hartmann, Jaffe, \& Huchra 1980; Altenhoff et al. 1981; Tanaka et al. 1985). It is believed that the massive, slow outflow originates in the surfaces of a neutral photoevaporating disk (Hollenbach et al. 1994; Lugo, Lizano, \& Garay 2004; Tafoya, Gómez, \& Rodríguez 2004). This disk has been inferred by: i) the presence of a double-peaked profile at optical, IR and radio recombination maser lines (Hamann \& Simon 1986; Planesas et al. 1992); ii) the rotation of the envelope around MWC 349A (Rodríguez \& Bastian 1994); iii) the presence of a dark lane in the equatorial plane of the source (White \& Becker 1985; Tafoya, Gómez \& Rodríguez 2004), and iv) observations in the near infrared that show a structure aligned in the direction of the equatorial dark lane (Danchi et al. 2001; Hofmann et al. 2002). The high angular resolution $7 \mathrm{~mm}$ observations (36 mas) made by Tafoya et al. (2004), show clearly the presence of a dark lane due to the lack of free-free emission from the neutral disk. These authors estimate a lower limit for the electron density

in the wind of $n_{e} \geq 1.4 \times 10^{7} \mathrm{~cm}^{-3}$ at a distance of $50 \mathrm{AU}$ from the star. This lower limit is consistent with the predicted electron density necessary to explain the H30 $\alpha$ maser recombination line (Martín-Pintado et al. 1989; Gordon 1992). MWC 349A is the only known source to exhibit maser amplification in its $\mathrm{mm}$ and sub-mm recombination lines.

The radio continuum emission from MWC 349A shows an extended bipolar structure with a waist that gets narrower as we observe at high frequencies (Tafoya et al. 2004). The radio spectrum is consistent with that expected for a biconical thermal wind that expands at constant velocity (e. g. Olnon 1975; Tafoya et al. 2004). The radio continuum emission also shows a faint structure to the west that suggests an interaction between the winds of MWC 349A and that of its companion MWC 349B (Cohen et al. 1985; Tafoya et al. 2004). MWC 349B has been classified as a B0 III star (Cohen et al. 1985).

Variability in the optical regime and in the millimeter maser recombination lines has been found toward MWC 349A (Bergner et al. 1995; Thum et al. 1992). In particular, Jorgenson et al. (2000) reported a red light curve covering the years 1967-1981, that suggests a period of $9 \mathrm{yr}$ and a probable amplitude variation of $\pm 0.4 \mathrm{mag}$. The study of Tafoya et al. (2004) suggested the presence of variation at $6 \mathrm{~cm}$ over the 14.5 years separating the two images analyzed. In this paper we report new 1.3, 2, and $6 \mathrm{~cm}$ observations as well as an extensive analysis of archive data to investigate possible time variations in more detail. 


\section{Observations}

The new 1.3, 2, and $6 \mathrm{~cm}$ observations were made in the A configuration of the VLA of the NRAO1, during 2004 November 15. The data were edited and calibrated following the standard VLA procedures. The absolute amplitude calibrator was $1331+305$ (with adopted flux densities of $2.52,3.46$, and $7.49 \mathrm{Jy}$ at $1.3,2$, and $6 \mathrm{~cm}$ respectively) and the phase calibrator was $2007+404$ (with bootstrapped flux densities of $3.20 \pm 0.11,3.18 \pm 0.02$, and $2.53 \pm 0.03 \mathrm{Jy}$ at $1.3,2$, and $6 \mathrm{~cm}$ respectively). The effective bandwidth of the observations was $100 \mathrm{MHz}$. All data were self-calibrated in phase.

For the discussion of time behavior of the source, we also used extensively data from the VLA archives, that are discussed in the following sections.

\section{Absolute Astrometry}

We used the four data sets obtained in the highest angular resolution A configuration at $2 \mathrm{~cm}$ that are listed in Table 1 to measure the proper motions of MWC 349A. These four data sets are appropriate for this determination since they span a large time baseline (1983.83 to 2004.87), have high angular resolution $(\sim 0.1)$, and were all taken with the same nearby phase calibrator, $2007+404$. The position used for $2007+404$ in the four epochs was updated to the most recent position given by the VLA calibrator manual, $\alpha(2000)=$ $20^{h} 07^{m} 44^{s} .944851 ; \delta(2000)=+40^{\circ} 29^{\prime} 48^{\prime \prime} 604140$. We fitted the position of MWC 349A with two techniques: 1) doing a least-squares fit of the source to a Gaussian ellipsoid using the task JMFIT of AIPS and 2) determining the offsets with respect to the 2004.87 epoch by minimizing the $\chi^{2}$ expression given by

$$
\chi^{2}=\sum_{x, y}\left[I\left(x-x_{o}, y-y_{o}\right)-I_{r e f}(x, y)\right]^{2}
$$

where $I$ is the intensity of the image being aligned, $I_{r e f}$ is the intensity of the reference image (in this case, the most recent image, taken in 2004.87), and $x_{o}$ and $y_{o}$ are the offsets. The sum is made over a rectangular box containing all the detectable emission from the source. The centroid position of the 2004.87 epoch image was determined to be $\alpha(2000)=$ $20^{h} 32^{m} 45.5284 ; \delta(2000)=+40^{\circ} 39^{\prime} 36^{\prime \prime} .623$ from the task JMFIT.

\footnotetext{
${ }^{1}$ The National Radio Astronomy Observatory is operated by Associated Universities Inc. under cooperative agreement with the National Science Foundation.
} 
Both methods gave consistent results and we finally used the $\chi^{2}$ method that gives the proper motions shown in Fig. 1. The positional errors at a given epoch were found to be of order 10 mas. A least-squares fit to these data gives average proper motions of:

$$
\begin{gathered}
\mu_{\alpha} \cos (\delta)=-3.1 \pm 0.5 \text { mas } y r^{-1} \\
\mu_{\delta}=-5.3 \pm 0.5 \text { mas }_{y^{-1}}
\end{gathered}
$$

To our knowledge, MWC 349A has no previously reported proper motions. Clemens \& Argyle (1984) tried to measure its proper motion from optical images, but their errors $\left(\sim 8\right.$ mas $\left.y r^{-1}\right)$ were about an order of magnitude larger than ours and this precluded a determination. The proper motions found by us for MWC 349A are similar to those measured by Hipparcos (Perryman et al. 1997) for the star P Cygni, $\mu_{\alpha} \cos (\delta)=-3.5 \pm$ 0.4 mas $\mathrm{yr}^{-1} ; \mu_{\delta}=-6.9 \pm 0.4$ mas $\mathrm{yr}^{-1}$. P Cygni is located at 3.9 in the plane of the sky from MWC 349A. We tried to use the determined proper motions of MWC 349A to set limits to its distance. Unfortunately, the proper motions in these galactic coordinates for objects at a few kpc from the Sun are relatively insensitive to the distance and we could not improve on what is known.

\section{4. $2 \mathrm{~cm}$ Flux Density as a Function of Time}

As discussed before, the results of Tafoya et al. (2004) suggested small variations, of order $1 \%$, between the two $6 \mathrm{~cm}$ images taken with a time separation of 14.5 years and analyzed by them.

We first analyzed if there is evidence in the archival data accumulated over the last two decades for significant variations in the total continuum flux density of MWC 349A. For this

analysis we used four data sets taken at $2 \mathrm{~cm}$ in the lowest angular resolution D configuration of the VLA. These observations of low angular resolution are more appropriate for measuring the total flux density of the source since they are less affected by the lack of short spacings or by phase noise produced by poor weather than those taken with higher angular resolution. In Table 2, we list the parameters of the observations and the flux densities determined. All values are consistent with a $2 \mathrm{~cm}$ flux density of $384 \pm 6 \mathrm{mJy}$, suggesting that the source has not suffered significant changes in its total $2 \mathrm{~cm}$ flux density over the last 20 years. This flux density is consistent at the $15 \%$ level with the power law fits given by Altenhoff et al. (1981) and Tafoya et al. (2004) to the continuum spectrum of MWC 349A. This lack of variability 
in the radio is previously known for MWC 349A, which allows its use as a flux calibrator in interferometers such as BIMA (e. g. Chen et al. 2006) and IRAM (e. g. Piétu et al. 2006). We will then assume that there is no variation in the total flux density of the source and for the morphology comparisons we will adjust the flux density scale of the observations made at different epochs to the flux density values given by the power law fit of Tafoya et al. (2004).

\section{Analysis of Morphology as a Function of Time}

\section{1. $2 \mathrm{~cm}$ Images}

For this analysis, we used the same four high angular resolution data sets used for the absolute astrometry (see Table 1). To allow an easier comparison, we made images with the same circular restoring beam of 0 "' 11 of half power full width. We consider this procedure adequate because the dimensions of the true synthesized beams of the observations differ only slightly from this value (see Table 1). These images are shown in Fig. 2. When comparing the images as a function of time, a remarkable variation is observed. While in 1983, the radio source had a well defined "waist" (that gave the source its "hourglass" shape) running approximately in the east-west direction, over the years this structure progressively disappeared, until by 2004 the source showed a more or less "square" morphology. These changes are also evident in the difference images, made from subtracting to the 2004 image the other epochs. In these difference images we see that the emission in the original north

and south lobes diminished with time while that in the "waist" of the nebula increased. We consider these results reliable since all four $2 \mathrm{~cm}$ databases used have similar $(\mathrm{u}, \mathrm{v})$ coverage, ranging from 40 to $1,500 \mathrm{k} \lambda$.

\section{2. $\quad 1.3 \mathrm{~cm}$ Images}

For this analysis, we used the three data sets presented in Table 3 . The $1.3 \mathrm{~cm}$ data is in general noisier than that at $2 \mathrm{~cm}$ and the variation is not as clear as at the latter wavelength. However, as can be seen in Fig. 3, the same trend is present in the $1.3 \mathrm{~cm}$ images, with the "waist" of the nebula disappearing with time. 


\section{3. $6 \mathrm{~cm}$ Images}

For this analysis, we used the three data sets presented in Table 4. Perplexingly, the time variation is different than that seen at 2 and $1.3 \mathrm{~cm}$ and consistent with the variation reported at $6 \mathrm{~cm}$ by Tafoya et al. (2004) using only the first two epochs (see Fig. 4). The new 2004 observations confirm the trend observed by Tafoya et al.. that is, the difference images clearly show a negative broken "ring" (with openings to the north and south) at a radius of $\sim 0.5$. This negative broken ring becomes more pronounced in the 2004.87-1982.42 image than in the 2004.87-1996.93, as expected for an effect that increases with time. The fact that the $6 \mathrm{~cm}$ images show a different behavior to that found in the 2 and $1.3 \mathrm{~cm}$ images is not in conflict with what is known of this source: in an ionized wind such as MWC 349A there is a radio "photosphere" that goes approximately as $\nu^{-0.7}$, implying that at $6 \mathrm{~cm}$ we are observing a region about 2 to 3 times larger that at 2 and $1.3 \mathrm{~cm}$. Then, the behaviors observed at different wavelengths do not have to coincide. In what remains of the paper we will concentrate our efforts in trying to account for the stronger morphology variations detected at 2 and $1.3 \mathrm{~cm}$.

\section{Interpretation}

\subsection{Precession of the MWC 349A Disk?}

One possibility is that the disk associated with MWC 349A has experienced significant precession over the 20 years of the study. In this possible explanation we will assume that the disk has suffered no intrinsic changes and that the observed differences are just a result of a change in orientation with respect to the observer.

To test if a precession of the disk could account for the changes, we made a model following the assumptions of White \& Becker (1985) and adding to the model the possibility of tilting the plane of the disk in the direction of the observer at a given inclination angle. This model is known to reproduce only approximately the appearance of the source. In particular, as emphasized by White \& Becker (1985), it does not reproduce the emission at large radii in the plane of the disk. Our comparison has then to be considered only qualitative. Nevertheless, analysis of Fig. 5 suggests that precession can indeed account qualitatively for the observed changes, in the sense that the nebula becomes progressively more square with increasing inclination and that from the point of view of the observer it is found that flux is "shifted" from the lobes to the plane of the disk.

However, is precession with such a small timescale (decades) possible? One could at- 
tribute the disk precession to the presence of the companion star MWC 349B. We will assume masses of $M_{p}=30 M_{\odot}$ for MWC 349A (the primary star) and of $M_{s}=18 M_{\odot}$ for $\mathrm{MWC}$ 349B (the secondary star), as estimated from the results of Rodríguez \& Bastian (1994) and Cohen et al. (1985). Assuming a distance of $1200 \mathrm{pc}$, we estimate that the stars are separated in the plane of the sky by a distance of $D=2900 A U$ and that the outer radius of the ionized disk at $2 \mathrm{~cm}$ is $R=360 A U$. Using the formulation of Terquem et al. (1999), the period of the precession of the disk (present around the primary star) is given by

$$
P_{p} \simeq 1.3 \times 10^{1}\left(\frac{M_{p}}{M_{s}}\right)\left(\frac{D}{R}\right)^{3}\left(\frac{R^{3}}{G M_{p}}\right)^{1 / 2},
$$

where $G$ is the gravitational constant. From the values given above, we estimate $P_{p} \simeq$ $2.3 \times 10^{6}$ years, far much larger that the timescale covered by the observations. We conclude that, although precession could explain the observed variations, the timescales expected from the effect of MWC 349B are just too large.

It is possible that if another star much closer to MWC349A is present, the precession could be faster. In the case of the source NGC 7538 IRS1, Kraus et al. (2006) present evidence of disk precession with a period of $\sim 280$ years, comparable with the timescales required for a precession model in MWC 349A. Kraus et al. (2006) propose as a possible triggering mechanism for precession in NGC 7538 IRS1 the non-coplanar tidal interaction of an (undiscovered) close companion with the circumbinary protostellar disk. Hofmann et al. (2002) have suggested that MWC 349A could be a close binary star, but there is no direct evidence for this. Furthermore, the estimated period for rotation of the outer radius of the

MWC 349A disk is of order $10^{3}$ years and it does not seem plausible to have precession scales on much shorter scales.

\subsection{Intrinsic Changes in the Disk?}

Another possibility to explain the observed changes is to have the disk itself experience significant changes. We do not know the structure and physical conditions of the disk with sufficient precision to model these possible changes and will only discuss if the expected timescales for variation are consistent with a few decades.

The kinematical timescale of the MWC 349A disk can be estimated by dividing the radius of the disk (360 AU) over the speed of the wind $\left(\sim 50 \mathrm{~km} \mathrm{~s}^{-1}\right)$, to obtain a timescale of about 35 years. A variation in this timescale could be related, for example, to the wind rapidly clearing the disk or to the photoevaporation occuring very rapidly. 
Another timescale of relevance is that given by the electron recombination time, which for an electron density of $10^{5} \mathrm{~cm}^{-3}$ (as expected in the outer parts of the disk) and an electron temperature of $10^{4} \mathrm{~K}$, gives about one year. A variation in this timescale could be related, for example, to the recombination or ionization of significant volumes of gas associated with the disk, presumably produced by changes in the central star.

Keto (2007, private communication) has used his model of a rotating accretion flow into a forming massive star (Keto 2007) to find that increases of order 10-30\% in the electron density of the flow can produce morphological changes that resemble the changes observed in MWC 349A over the last two decades.

We conclude that, based on the timescales involved, the possibility on intrinsic changes in the disk seems more plausible than that of precession. To obtain a qualitative impression of the appearance changes expected from changes in the disk, we have again used the model of White \& Becker (1985) and, for an inclination angle of $10^{\circ}$ with respect to the observer, we have varied the critical angle $\theta_{c}$ above which the gas is fully ionized. The critical angle is equivalent to the flaring angle of the disk. These variations could be produced by very rapid photoevaporation of the surface of the disk. As can be seen in Fig. 6, again we have that flux from the lobes "moves" to the waist of the nebula and the appearance becomes squared, as we diminish $\theta_{c}$.

However, as in the case of precession, the expected timescales for significant photoevaporation of the disk are much larger than a few decades. The mass of the MWC 349A disk is not known, but Danchi et al. (2001) give upper limits of a few solar masses. Tafoya et al. (2004) give a mass loss rate in ionized gas of $5.0 \times 10^{-6} M_{\odot} y r^{-1}$. Assuming a mass of $1 M_{\odot}$ for the disk, we find a photoevaporation timescale of $2 \times 10^{5} \mathrm{yr}$. An even larger timescale, of several million years, is given by Yorke \& Weltz (1996) from models of the evolution of photoevaporating disks around early B type stars.

\section{Conclusions}

We have analyzed new as well as archive continuum VLA data of the peculiar emissionline star MWC 349A covering more than 20 years of time. We determined for the first time the proper motions of this source, which are consistent with those expected for its location in the galaxy.

The appearance of MWC 349 has been systematically changing over time and we discuss if those variations are due to precession of the disk or to intrinsic changes in the source. Although these types of variations produce changes qualitatively similar to those found in 
the observations, the timescales expected for them are much larger than the two decades comprised in the observations. We could not reach a satisfactory explanation for the varia-

tions and additional detailed theoretical modeling and continuous monitoring are needed to advance in our understanding of this source.

LFR and YG acknowledge the support of CONACyT, México and DGAPA, UNAM. Facilities: VLA

\section{REFERENCES}

Altenhoff, W. J., Strittmatter, P. A., \& Wendker, H. J. 1981, A\&A, 93, 48

Bergner, Y. K., Miroshnichenko, A. S., Yudin, R. V., Kuratov, K. S., Mukanov, D. B., \& Shejkina, T. A. 1995, A\&AS, 112, 221

Braes, L. L. E., Habing, H. J., \& Schoenmaker, A. A. 1972, Nature, 240, 230

Chen, H.-R., Welch, W. J., Wilner, D. J., \& Sutton, E. C. 2006, ApJ, 639, 975

Clemens, E. D. \& Argyle, R. W. 1984, MNRAS, 209, 1

Cohen, M., Bieging, J. H., Dreher, J. W., \& Welch, W. J. 1985, ApJ, 292, 249

Danchi, W. C., Tuthill, P. G., \& Monnier, J. D. 2001, ApJ, 562, 440

Hamann, F., \& Simon, M. 1986, ApJ, 311, 909

Hartmann, L., Jaffe, D., \& Huchra, J. P. 1980, ApJ, 239, 905

Hofmann, K.-H., Balega, Y., Ikhsanov, N. R., Miroshnichenko, A. S., \& Weigelt, G. 2002, A\&A, 395, 891

Hollenbach, D., Johnstone, D., Lizano, S., \& Shu, F. 1994, ApJ, 428, 654

Gordon, M. A. 1992, ApJ, 387, 701

Jorgenson, R. A., Kogan, L. R., \& Strelnitski, V. 2000, AJ, 119, 3060

Keto, E. R. 2007, submitted to ApJ, astro-ph/0603856

Kraus, S., Balega, Y., Elitzur, M., Hofmann, K.-H., Preibisch, Th., Rosen, A., Schertl, D., Weigelt, G., \& Young, E. T. 2006, A\&A, 455, 521 
Lugo, J., Lizano, S., \& Garay, G. 2004, ApJ, 614, 807

Martín-Pintado, J., Bachiller, R., Thum, C., \& Walmsley, M. 1989, A\&A, 215, L13

Olnon, F. M. 1975, A\&A, 39, 217

Perryman, M. A. C. et al. 1997, A\&A, 323, L49

Piétu, V., Dutrey, A., Guilloteau, S., Chapillon, E., \& Pety, J. 2006, A\&A, 460, L43

Planesas, P., Martín-Pintado, J., \& Serabyn, E. 1992, ApJ, 386, L23

Rodríguez, L. F., \& Bastian, T. S. 1994, ApJ, 428, 324

Tafoya, D., Gómez, Y., \& Rodríguez, L. F. 2004, ApJ, 610, 827

Tanaka, M., Yamashita, T., Sato, S., Nishida, M., Ukita, N., \& Okuda, H. 1985, PASP, 97, 1115

Terquem, C., Eislöffel, J., Papaloizou, J. C. B., \& Nelson, R. P. 1999, ApJ, 512, L131

Thum, C., Martín-Pintado, J., \& Bachiller, R. 1992, A\&A, 256, 507

White, R. L., \& Becker, R. H. 1985, ApJ, 297, 677

Yorke, H. W., \& Welz, A, 1996, A\&A, 315, 555 

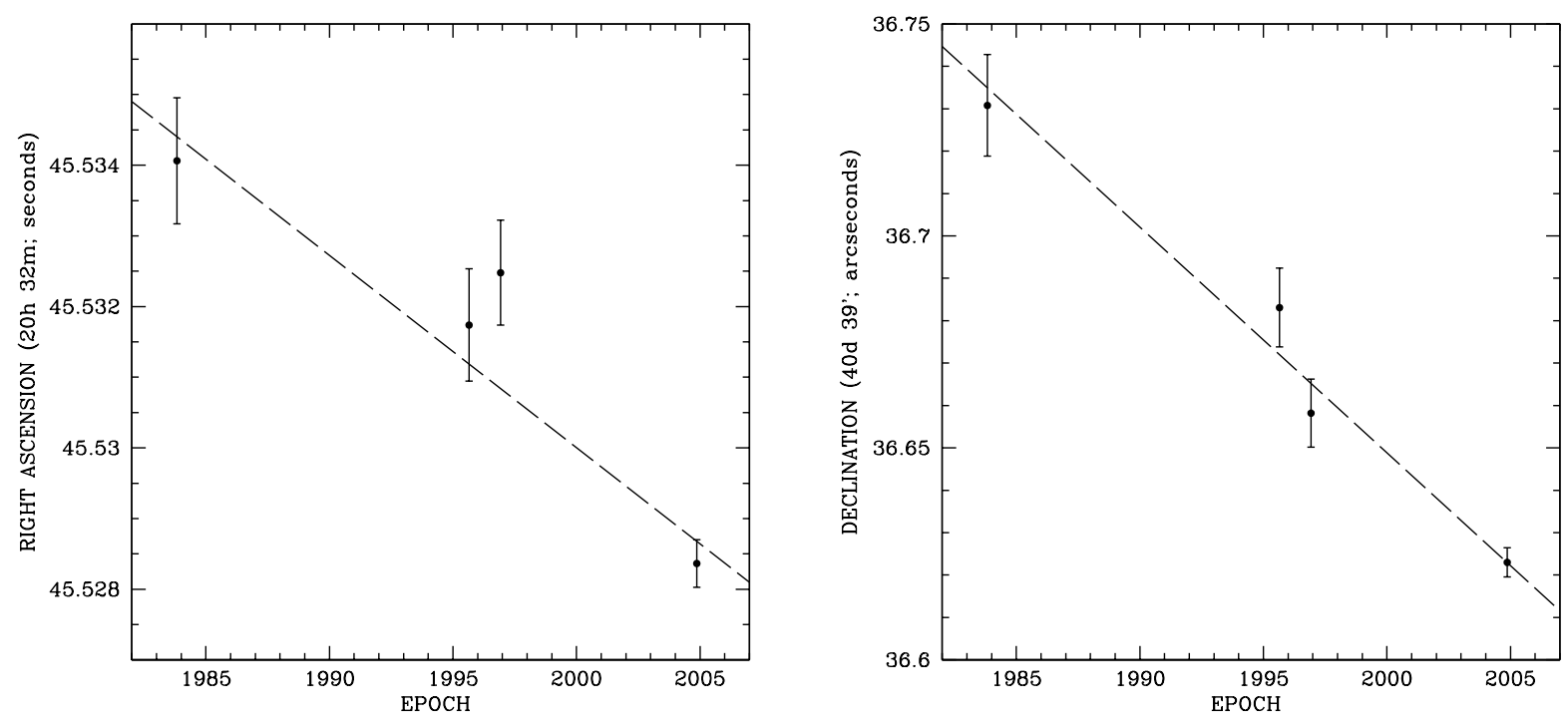

Fig. 1.- Positions in right ascension (left) and declination (right) for MWC 349A as a function of epoch. These positions are obtained from the four epochs listed in Table 1. The dashed lines are the least-squares fits to the data, that give proper motions $\mu_{\alpha} \cos (\delta)=$ $-3.1 \pm 0.5$ mas $_{y r^{-1}} ; \mu_{\delta}=-5.3 \pm 0.5$ mas $r^{-1}$. 

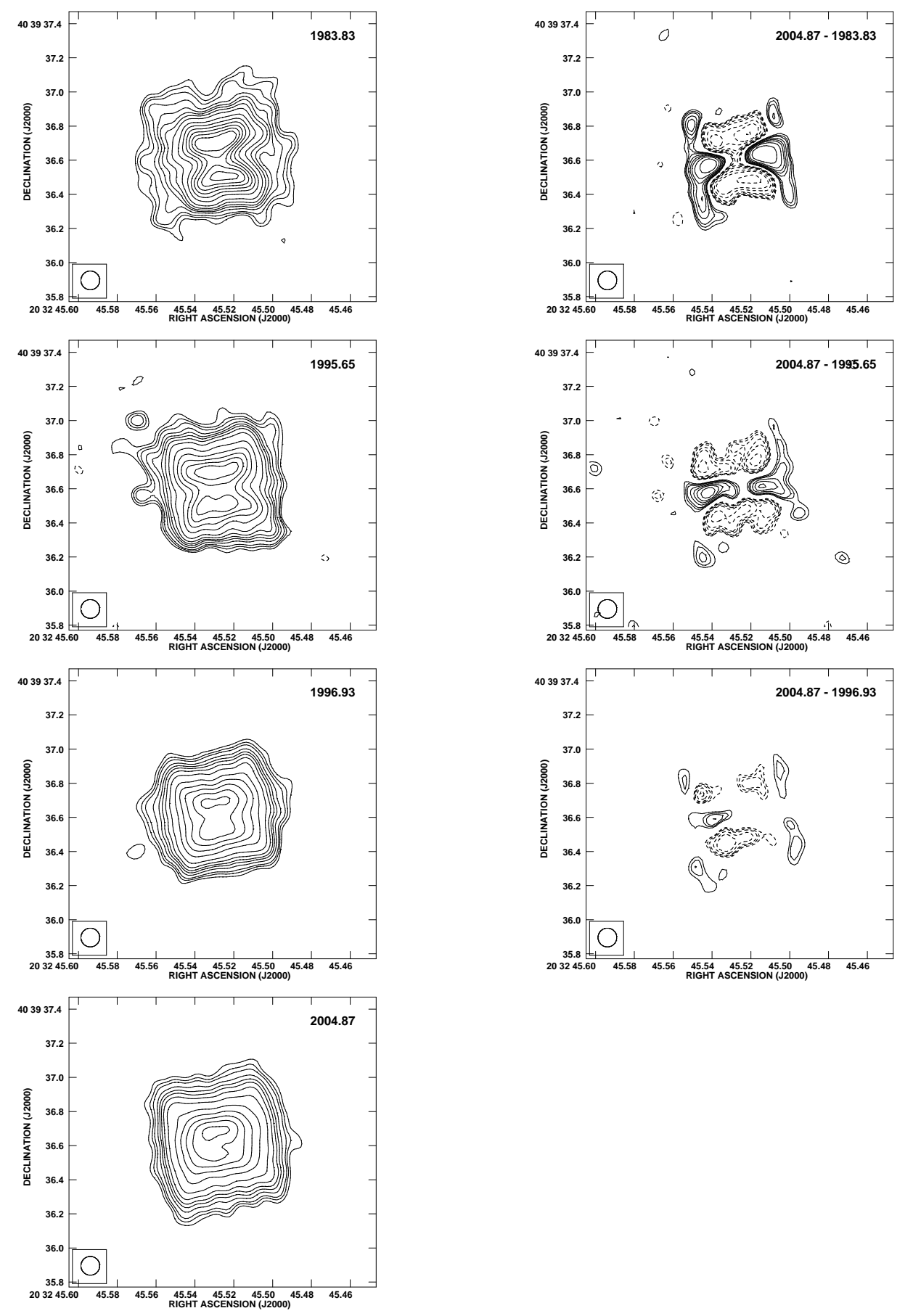

Fig. 2.- (Left) Contour images of the $2 \mathrm{~cm}$ emission from MWC 349A for four different epochs. (Right) Contour plots of the $2 \mathrm{~cm}$ difference images. Contours are $-20,-15,-12,-10$, $-8,-6,-5,-, 4,4,5,6,8,10,12,15,20,30,40,50,60,70$, and 80 times $0.30 \mathrm{mJy} \mathrm{beam}^{-1}$. The half power contour of the restoring beam $(0.11 \times 0 . \prime 11)$ is shown in the bottom left corner. The images of the first three epochs are corrected for proper motion and aligned to the 2004.87 position of MWC 349A. 

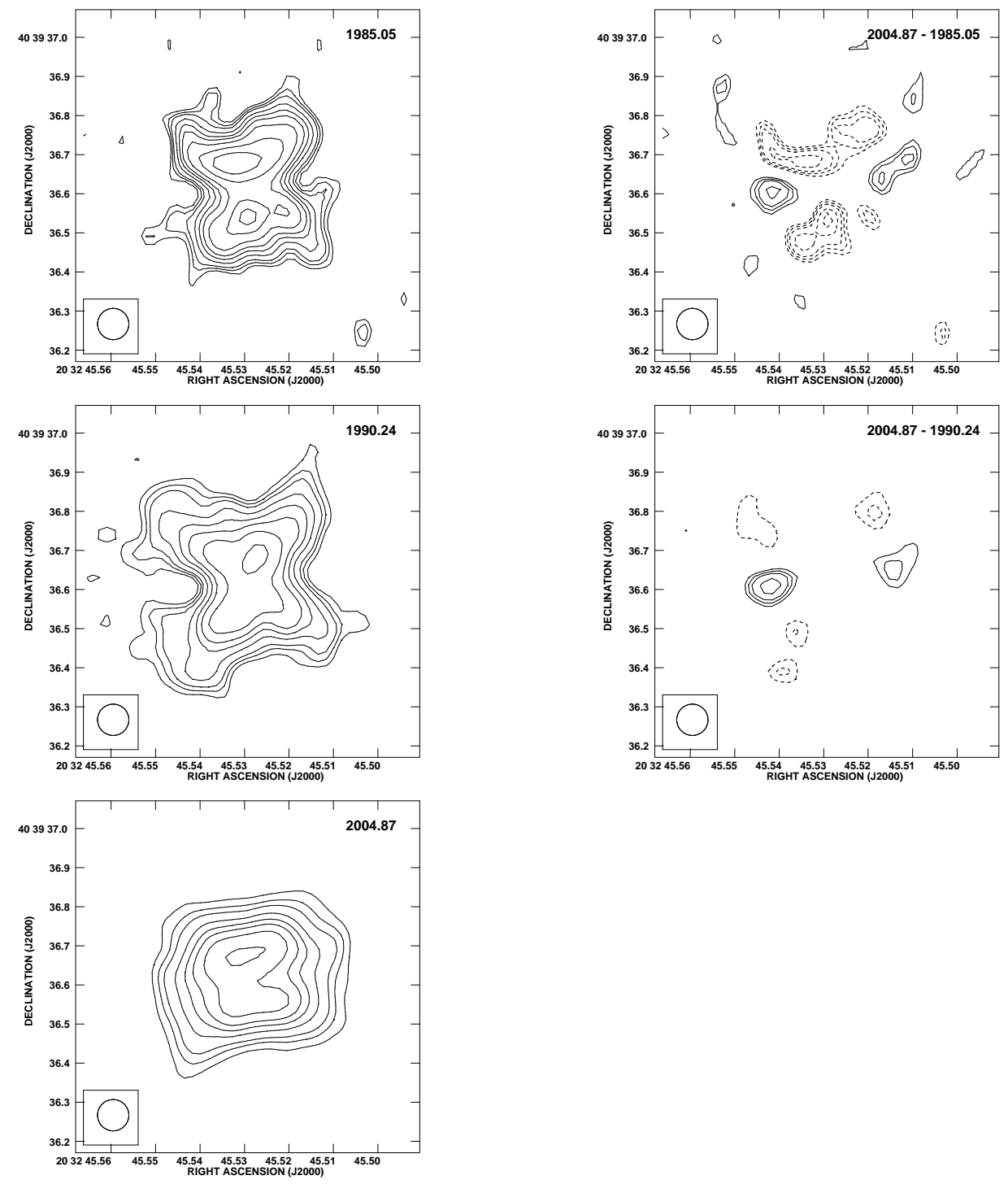

Fig. 3.- (Left) Contour images of the $1.3 \mathrm{~cm}$ emission from MWC 349A for three different epochs. (Right) Contour plots of the $1.3 \mathrm{~cm}$ difference images. Contours are $-10,-8,-6$, $-5,-, 4,4,5,6,8,10,12,15,20$, and 25 times $1.2 \mathrm{mJy}_{\text {beam }}{ }^{-1}$. The half power contour of the restoring beam $\left(00^{\prime \prime} 08 \times 0{ }^{\prime \prime} 08\right)$ is shown in the bottom left corner. The images of the first three epochs are corrected for proper motion and aligned to the 2004.87 position of MWC 349A. 

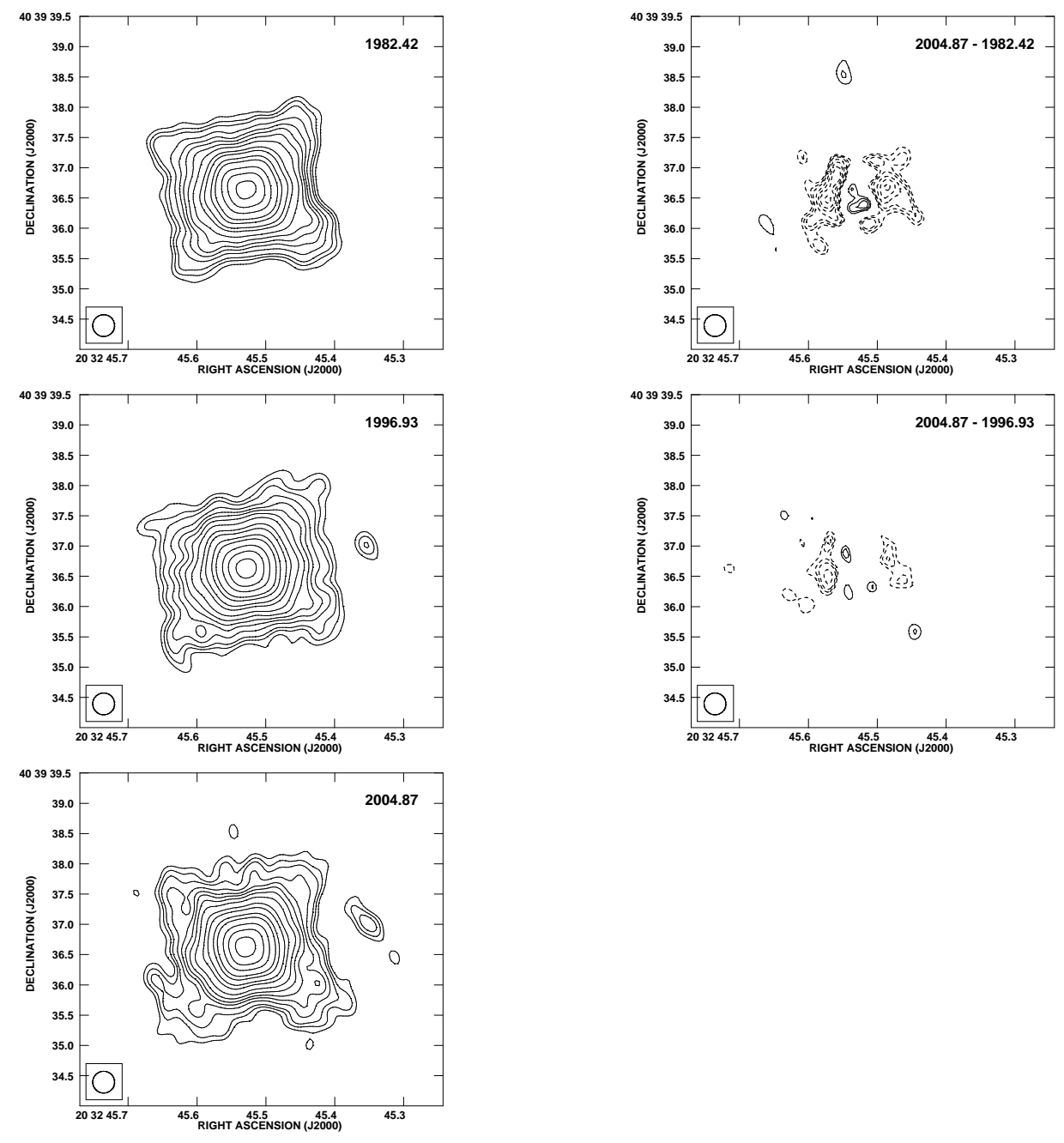

Fig. 4.- (Left) Contour images of the $6 \mathrm{~cm}$ emission from MWC 349A for three different epochs. The feature to the west of MWC 349A is the interaction zone of the stellar winds located between MWC 349A and MWC 349B that is discussed in detail by Tafoya et al. (2004). (Right) Contour plots of the $6 \mathrm{~cm}$ difference images. Contours are -20, -15, -12, $-10,-8,-6,-5,-, 4,4,5,6,8,10,12,15,20,30,40,60,80,120,160$, and 200 times $0.10 \mathrm{mJy}$ beam $^{-1}$. The half power contour of the restoring beam $\left(0{ }^{\prime \prime} 36 \times 0\right.$ "'36) is shown in the bottom left corner. The images of the first three epochs are corrected for proper motion and aligned to the 2004.87 position of MWC 349A. 

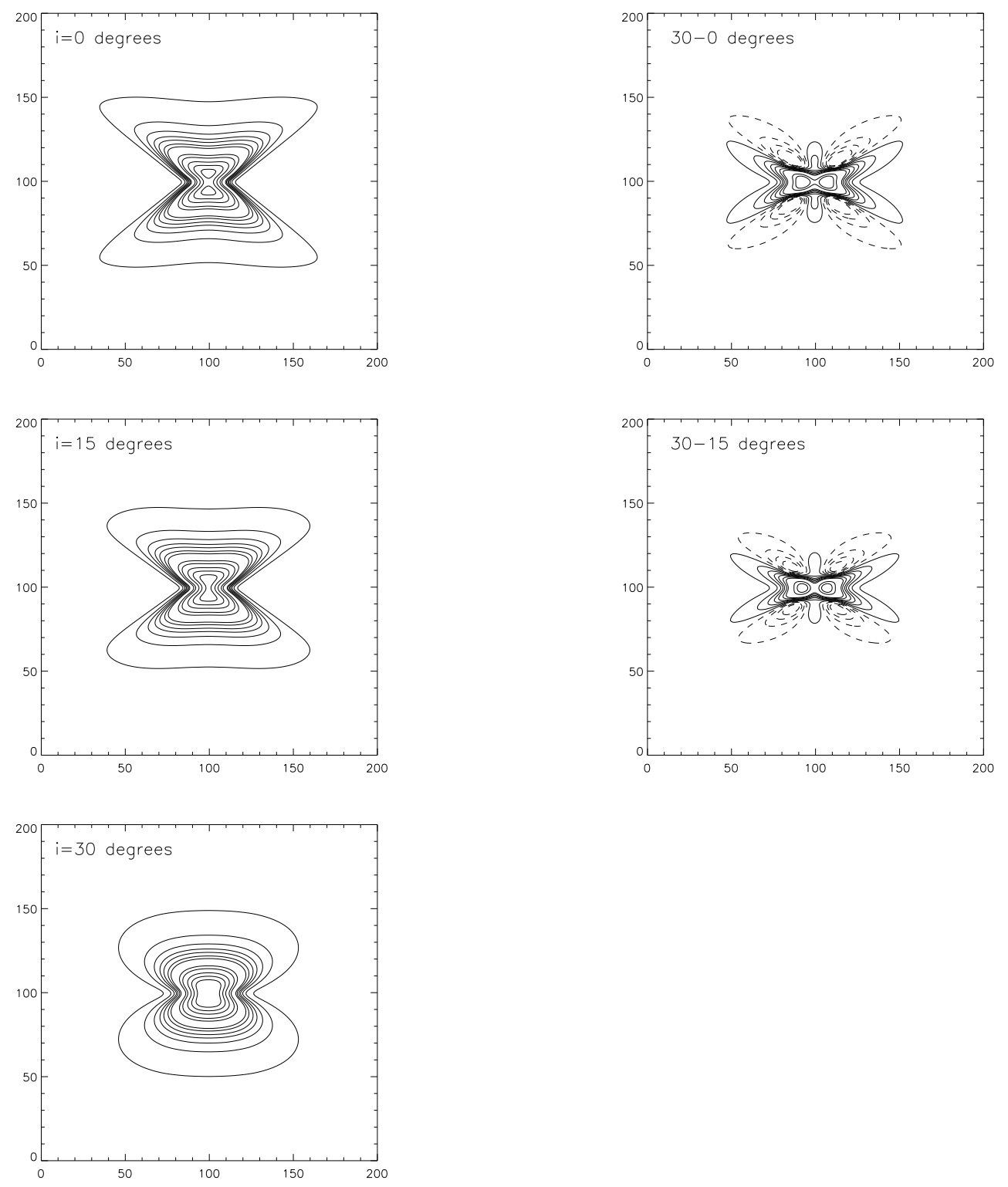

Fig. 5.- (Left) Contour images of the precession model described in the text. The inclination angle of the plane of the disk of the model with respect to the line of sight is indicated in each figure. (Right) Contour plots of the model difference images. Contours are -100,-80, $-60,-40,-30,-15,-12,-9,-7,-5,-3,-1,1,3,5,7,9,12,15,30,40,60,80$, and 100 percent the peak value of the model with inclination angle of $0^{\circ}$. The $x$ and $y$ axes of the models are in units of 0 .'01 and the images are smoothed to an angular resolution of 0.1 . 

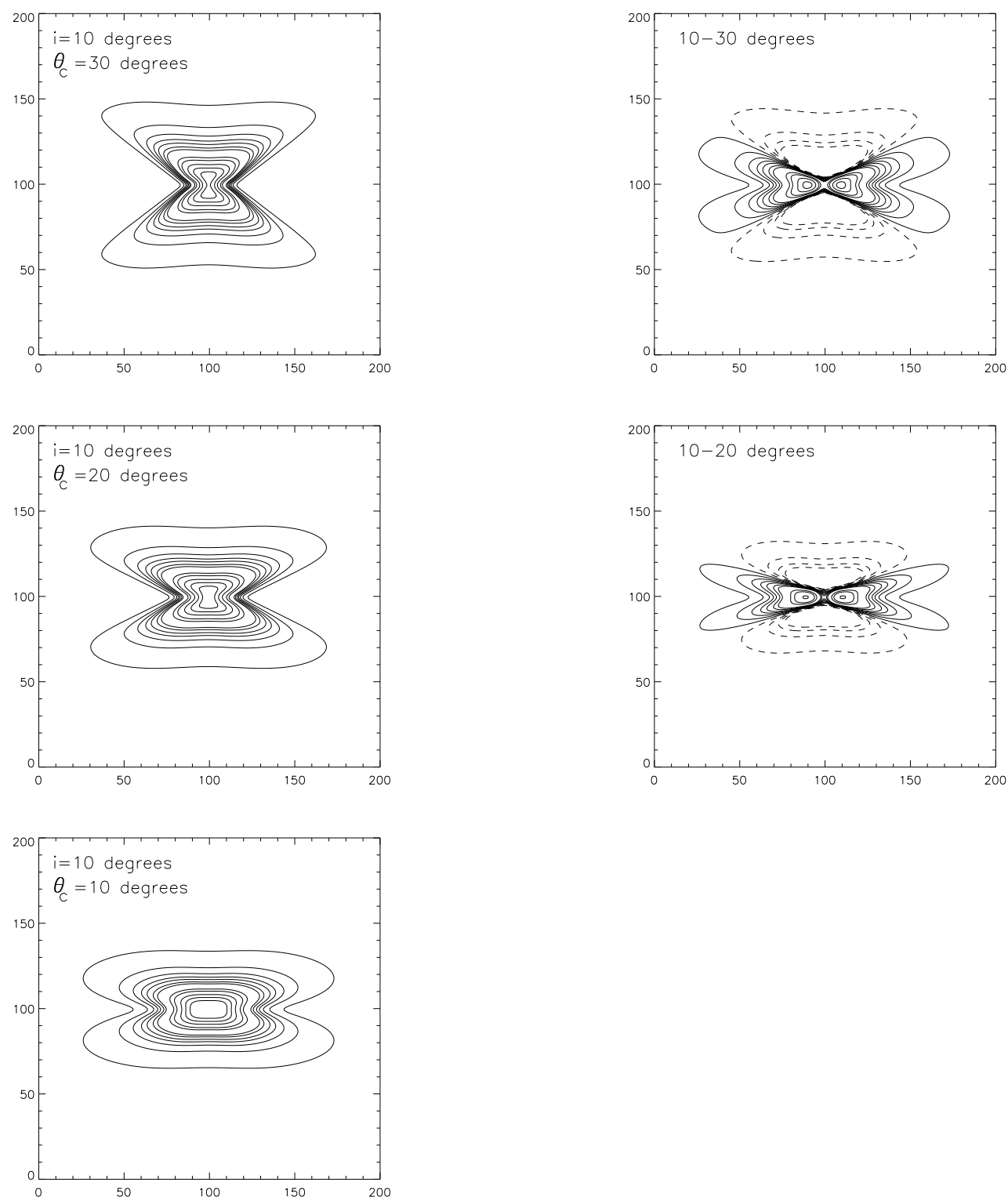

Fig. 6.- (Left) Contour images of the model with variable critical angle $\theta_{c}$ described in the text. The inclination angle of the plane of the disk of the model with respect to the line of sight is always $10^{\circ}$ and the critical angles are indicated in each figure. (Right) Contour plots of the model difference images. The differences refer to the critical angle $\theta_{c}$. Contours are $-100,-80,-60,-40,-30,-15,-12,-9,-7,-5,-3,-1,1,3,5,7,9,12,15,30,40,60,80$, and 100 percent the peak value of the model with critical angle of $30^{\circ}$. The $x$ and $y$ axes of the models are in units of $0 . .01$ and the images are smoothed to an angular resolution of 0. . 1 . 
Table 1. Configuration A Observations at $2 \mathrm{~cm}$ Analyzed

\begin{tabular}{|c|c|c|}
\hline Epoch & $\begin{array}{c}\text { Phase } \\
\text { Calibrator }\end{array}$ & $\begin{array}{l}\text { Synthesized Beam } \\
\qquad\left(\theta_{M} \times \theta_{m} ; P A\right)^{\mathrm{a}}\end{array}$ \\
\hline 1983 Oct 30 (1983.83) & $2007+404$ & $0^{\prime \prime} 12 \times 0^{\prime \prime} .10 ;+5^{\circ}$ \\
\hline 1995 Aug 24 (1995.65) & $2007+404$ & $0.13 \times 0 . .11 ;+30^{\circ}$ \\
\hline 1996 Dec 05 (1996.93) & $2007+404$ & $0.12 \times 0 . .11 ;-32^{\circ}$ \\
\hline 2004 Nov 15 (2004.87) & $2007+404$ & $0^{\prime \prime} 12 \times 0^{\prime \prime} 11 ;+89^{\circ}$ \\
\hline
\end{tabular}

${ }^{a}$ Major axis $\times$ minor axis in arcsec; PA in degrees for synthesized beam made with ROBUST $=0$. 
Table 2. Flux Densities from Configuration D Observations at $2 \mathrm{~cm}$

\begin{tabular}{cccc}
\hline \hline Epoch & $\begin{array}{c}\text { Phase } \\
\text { Calibrator }\end{array}$ & $\begin{array}{c}\text { Synthesized Beam } \\
\left(\theta_{M} \times \theta_{m} ; P A\right)^{\mathrm{a}}\end{array}$ & $\begin{array}{c}\text { Flux Density } \\
\text { of MWC 349A (mJy) }\end{array}$ \\
\hline 1984 Aug 19 (1984.63) & $2007+404$ & $4^{\prime \prime} .3 \times 3^{\prime \prime \prime} 9 ;-89^{\circ}$ & $390 \pm 30$ \\
1996 Jul 30 (1996.58) & $2007+404$ & $4^{\prime \prime} 4 \times 3^{\prime \prime} 8 ;+11^{\circ}$ & $383 \pm 20$ \\
2001 Nov 09 (2001.86) & b & $5^{\prime \prime} 3 \times 4^{\prime \prime} .3 ;-90^{\circ}$ & $380 \pm 6$ \\
2006 Jan 20 (2006.05) & $\mathrm{b}$ & $9^{\prime \prime} 4 \times 5^{\prime \prime} .4 ;-55^{\circ}$ & $378 \pm 15$ \\
\hline
\end{tabular}

a Major axis $\times$ minor axis in arcsec; PA in degrees for synthesized beam made with $\operatorname{ROBUST}=0$.

bThese observations were made without a phase calibrator and the flux calibration was transferred directly from $1331+305$, the amplitude calibrator, to MWC 349A. 
Table 3. Configuration A Observations at $1.3 \mathrm{~cm}$ Analyzed

\begin{tabular}{|c|c|c|}
\hline Epoch & $\begin{array}{c}\text { Phase } \\
\text { Calibrator }\end{array}$ & $\begin{array}{l}\text { Synthesized Beam } \\
\qquad\left(\theta_{M} \times \theta_{m} ; P A\right)^{\mathrm{a}}\end{array}$ \\
\hline 1985 Jan 20 (1985.05) & $2007+404$ & $0 . \prime 08 \times 0 . .07 ;-22^{\circ}$ \\
\hline 1990 Mar 29 (1990.24) & $2007+404$ & $0.09 \times 0 . .08 ;-68^{\circ}$ \\
\hline 2004 Nov 15 (2004.87) & $2007+404$ & $0 . .09 \times 0 . .07 ;-85^{\circ}$ \\
\hline
\end{tabular}

${ }^{\text {a }}$ Major axis $\times$ minor axis in arcsec; PA in degrees for synthesized beam made with ROBUST $=0$. 
Table 4. Configuration A Observations at $6 \mathrm{~cm}$ Analyzed

\begin{tabular}{ccc}
\hline \hline Epoch & $\begin{array}{c}\text { Phase } \\
\text { Calibrator }\end{array}$ & $\begin{array}{c}\text { Synthesized Beam } \\
\left(\theta_{M} \times \theta_{m} ; P A\right)^{\mathrm{a}}\end{array}$ \\
\hline 1982 Jun 04 (1982.42) & $2007+404$ & $0^{\prime \prime} 37 \times 0 . \prime 34 ;+2^{\circ}$ \\
1996 Dec 05 (1996.93) & $2007+404$ & $0^{\prime \prime} 38 \times 0^{\prime \prime \prime} 34 ;-9^{\circ}$ \\
2004 Nov 15 (2004.87) & $2007+404$ & $0^{\prime \prime} 38 \times 0^{\prime \prime} 34 ;-80^{\circ}$ \\
\hline
\end{tabular}

${ }^{a}$ Major axis $\times$ minor axis in arcsec; PA in degrees for synthesized beam made with ROBUST $=0$. 